\title{
Trace metal distribution in muddy-patch sediments from the northern Portuguese Shelf
}

\author{
Distribución de metales traza en sedimentos de zonas de lodo \\ de la plataforma costera norte de Portugal
}

\author{
Maria-João Madureira \\ Carlos Vale* \\ Nuno Fonseca \\ Institute for Fisheries and Sea Research (IPIMAR) \\ Av. Brasília, 1449-006 Lisboa, Portugal \\ *E-mail: cvale@ipimar.pt
}

Recibido en diciembre de 2001; aceptado en octubre de 2002

\begin{abstract}
Three sediment cores were collected in the muddy patches of the northern Portuguese Shelf located offshore the Douro and Minho rivers. Bivalves and worms were found in the upper and sub-surface layers of two cores. Vertical distributions of dissolved manganese and iron, iron oxides and acid volatile sulphide (AVS) in the bioturbated cores differ dramatically from those in the core where macrofauna was not observed. Also, $\mathrm{Fe}, \mathrm{Zn}, \mathrm{Cu}, \mathrm{Pb}$ and $\mathrm{Cd}$ simultaneously extracted with AVS (1 M HCl) showed pronounced increases in concentrations deeper in the sediments. Where bioturbation was not visible the profiles of extractable $\mathrm{Cu}, \mathrm{Pb}$ and $\mathrm{Cd}$ are consistent with the precipitation of downward diffusing dissolved metals. However, this redistribution did not affect the metal-total vertical profiles. Reactive metals exhibited multiple peaks in the irrigated sub-surface layers where AVS and dissolved Fe showed successive maxima and minima with the depth. We interpreted these profiles as the net result of dissolved oxygen being pumped into the sediment and the formation of several oxic/anoxic interfaces. At these depths trace metals are mobilized in the oxidized layers, diffused a few millimetres towards the layers where sulphide is present, and precipitated. The consequence of this internal redistribution was the broad maxima of total metal concentrations in irrigated sediment depths.
\end{abstract}

Key words: trace metals, sulphides, early diagenesis, northern Portuguese Shelf.

\section{Resumen}

Se muestrearon tres testigos de sedimento en manchas de lodo procedentes de la plataforma continental del norte de Portugal localizadas frente a los ríos Duero y Miño. En las capas superficial y subsuperficial de dos de los testigos se encontraron bibalvos y gusanos. Las distribuciones verticales de hierro y manganeso disueltos, óxidos de hierro y sulfuros volátiles ácidos (AVS) en los testigos bioperturbados son drásticamente diferentes de aquellos en los que no se observó macrofauna alguna. De igual forma, el $\mathrm{Fe}, \mathrm{Zn}, \mathrm{Cu}, \mathrm{Pb}$ y Cd extraídos simultáneamente con los AVS ( $\mathrm{HCl} 1 \mathrm{M})$, mostraron incrementos marcados en la concentración en zonas más profundas del sedimento. Donde la bioperturbación no fue visible, los perfiles de $\mathrm{Cu}, \mathrm{Pb}$ y $\mathrm{Cd}$ extraíbles concuerdan con la precipitación y los metales disueltos con difusión descendente. Sin embargo, esta redistribución no afectó los perfiles verticales de metales totales. Los metales reactivos mostraron picos múltiples en capas irrigadas sub-superficiales donde los AVS y el Fe disuelto presentaron máximos y mínimos sucesivos con la profundidad. Interpretamos estos perfiles como el resultado neto del bombeo de oxígeno disuelto hacia el sedimento y la formación de varias interfases óxicas-anóxicas. En estas profundidades, los metales traza son movilizados en las capas oxidadas, y difundidos unos pocos milímetros hacia las capas donde hay sulfuro y éste precipita. La consecuencia de esta redistribución interna determina el máximo de las concentraciones totales de metales en profundidades con sedimentos irrigados.

Palabras clave: metales traza, sulfuros, diagenesis temprana, plataforma norte de Portugal.

\section{Introduction}

The interpretation of the sedimentary metal record requires a clear understanding of metal mobility and preservation during the early diagenesis of sediments (Berner, 1980). Several works have shown evidence of the release of transition metals associated with the degradation of biogenic detritus in oxic/suboxic sediment layers (Klinkhammer et al., 1982; Gobeil et al., 1987; Gobeil and Silverberg, 1989; Shaw et al.,

\section{Introducción}

La interpretación del registro sedimentario de metales requiere una comprensión clara de su movilidad y preservación durante la diagénesis temprana de los sedimentos (Berner, 1980). Varios trabajos han dado evidencia de la liberación de metales de transición asociados con la degradación de desechos biogénicos en capas óxicas/subóxicas del sedimento (Klinkhammer et al., 1982; Gobeil et al., 1987; Gobeil y 
1990). The concentration-depth profiles are maintained by a coupling of this regeneration with varying degrees of interaction between dissolved metal and sediment below the interface (Klinkhammer et al., 1982). Most of the well-defined profiles were registered in marine sediment deposits consisting of sharply defined zones, within which only certain redox reactions can occur (Froelich et al., 1979). In coastal sediments, the vertical chemical zonation in both pore water and solid fraction tends to be less clear, due to the presence and activity of benthic organisms (Aller, 1977) and seasonal variations in organic carbon input to the sediment and in temperature (Aller, 1994; Thamdrup et al., 1994). These variations can cause a fluctuation in the vertical position of the oxic/anoxic boundary within the sediment, and create zones where metals are alternately oxidized and reduced, and consequently, metal sulphides are alternately precipitated and dissolved (Gobeil et al., 1997; Brugmann et al., 1998). Modifications have also been reported in deep-sea sediments due to an overall change in the ocean productivity during the last millennium (Pedersen et al., 1986) and during the sapropel formation in the North Atlantic (Thomson et al., 1993).

The muddy patches of Douro and Minho are located in the NW Iberian Margin, around $20 \mathrm{~km}$ northwest offshore the Douro and Minho rivers, respectively. The deposit of fine particles transported from the continent is facilitated by the non-uniform topography of the area (Dias and Nittrouer, 1984). The sedimentation rate in the area was estimated between 0.1 and $0.3 \mathrm{~cm} /$ year (Drago et al., 1998). Elemental analyses of upper sediment layers revealed no relationships with the fluvial sources and relatively uniform metal concentrations (Araújo et al., 1994; Barbosa et al., 1999). Postdepositional changes in the sediment chemical composition have not been investigated. In this paper we present data on the distribution of copper, lead, cadmium, manganese and iron in sediment cores from the Douro and Minho muddy patches. In some cores, maxima of reactive and total trace metals were registered in sub-surface layers where sulphide concentrations changed pronouncedly. We describe the relationships between iron, manganese and trace metal distribution and discuss the possibility of the vertical distributions reflecting changes in the redox conditions within the sediment.

\section{Material and methods}

\section{Sampling}

Three sediment cores were sampled in two muddy patches located offshore the Douro and Minho rivers, in the northern Portuguese Shelf (fig. 1). The samples were collected using a multi-corer aboard the research vessel Mestre Costeiro in 1998. Cores E and G, $30 \mathrm{~cm}$ long, were collected from the Douro muddy patch and core J, $15 \mathrm{~cm}$ long, from the Minho patch. The cores, not disturbed by the sampling, were subsampled on board into $0.5-\mathrm{cm}$ horizontal layers in the first $10 \mathrm{~cm}$ and then in $1-\mathrm{cm}$ intervals. Deeper sediments were
Silverberg, 1989; Shaw et al., 1990). Los perfiles de concentración-profundidad se mantienen por un acoplamiento de esta regeneración con varios grados de interacción entre los metales disueltos y el sedimento por debajo de la interfase (Klinkhammer et al., 1982). La mayoría de los perfiles bien definidos fueron registrados en depósitos de sedimentos marinos que consistían en zonas perfectamente establecidas dentro de las cuales sólo ciertas reacciones de tipo redox pueden ocurrir (Froelich et al., 1979). La zonificación química vertical tanto en agua intersticial como en la fracción sólida de los sedimentos costeros tiende a ser menos clara, debido a la presencia y actividad de organismos bénticos (Aller, 1977), y a variaciones estacionales en el aporte de carbono orgánico al sedimento y la temperatura (Aller, 1994; Thamdrup et al., 1994). Estas variaciones pueden originar una fluctuación en la posición vertical de la frontera óxico-anóxica dentro del sedimento, crear zonas en las que los metales se encuentran oxidados y reducidos de manera alternada y, en consecuencia, los sulfuros metálicos se encuentran precipitados o disueltos también alternadamente (Gobeil et al., 1997; Brugmann et al., 1998). Estas modificaciones también han sido reportadas en sedimentos marinos profundos debido a un cambio total en la productividad oceánica durante el último milenio (Pedersen et al., 1986) y durante la formación de cieno rico en materia orgánica en el Atlántico Norte (Thomson et al., 1993).

Las zonas de lodo del Duero y del Miño se localizan al noroeste del margen ibérico, alrededor de $20 \mathrm{Km}$ al noroeste de las desembocaduras de los ríos Duero y Miño, respectivamente. La depositación de las partículas finas transportadas desde el continente es facilitada por la topografía irregular del área (Dias y Nittrouer, 1984). La tasa de sedimentación fue estimada entre 0.1 y $0.3 \mathrm{~cm} /$ año (Drago et al., 1998). Los análisis elementales de las capas superficiales del sedimento no revelaron relación alguna entre las fuente fluviales y las concentraciones de metales relativamente uniformes (Araujo et al., 1994; Barbosa et al., 1999). Los cambios en la composición química del sedimento después de la depositación no han sido investigados aún. En este documento presentamos datos acerca de la distribución del cobre, plomo, cadmio, manganeso y hierro en testigos de sedimentos de las zonas de lodo del Duero y del Miño. En algunos testigos se registraron máximos de metales traza, tanto reactivos como totales, en las capas subsuperficiales donde las concentraciones de sulfuro cambiaron abruptamente. También describimos la relación entre las distribuciones de hierro, manganeso y metales traza, y discutimos acerca de la posibilidad de que las distribuciones verticales reflejen cambios en las condiciones redox del sedimento.

\section{Material y métodos}

\section{Muestreo}

Se muestrearon tres testigos de sedimento en dos zonas de lodo localizados frente a los ríos Duero y Miño en la plataforma costera del norte de Portugal (fig. 1), utilizando un 


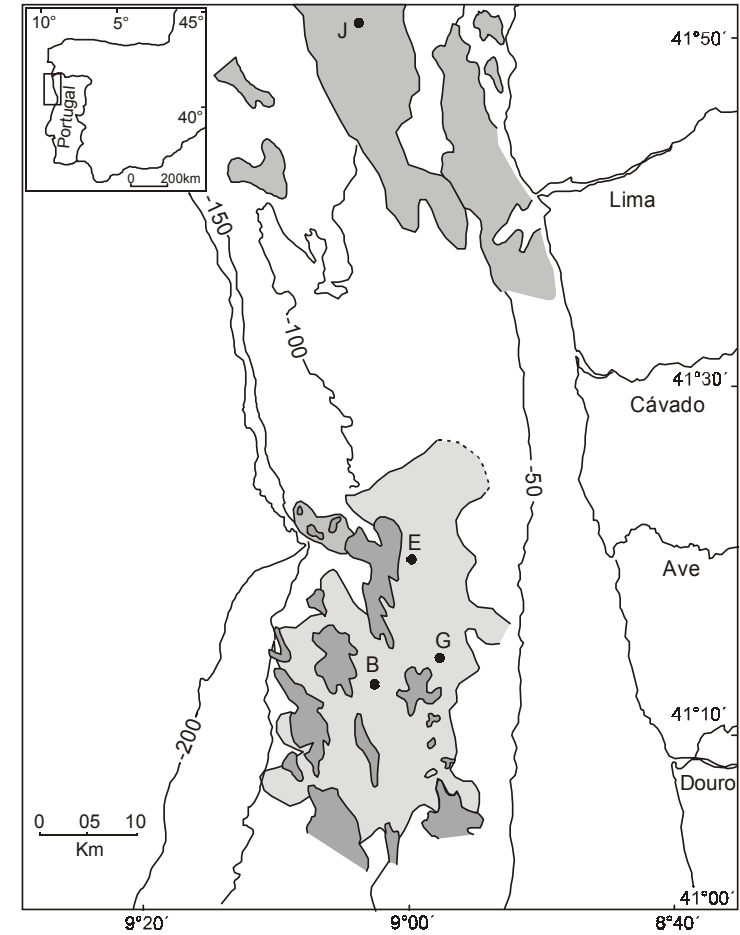

Figure 1. Map of the Douro and Minho muddy patches with the location of stations $E, G$ and J.

Figura 1. Mapa de las zonas de lodo del Duero y del Miño con la localización de las estaciones $\mathrm{E}, \mathrm{G}$ y J.

sectioned in 2-cm layers. A 1-g sediment aliquot was separated immediately for sulphide and amorphous iron determinations. All these operations were done quickly in order to minimize changes resulting from the exposure of the sediment slices to the atmosphere. Samples were stored in leakproof polycarbonate tubes, completely filled in a glove box under nitrogen, and kept in a refrigerated chamber. In the laboratory, samples were centrifuged for $30 \mathrm{~min}$ at $3000 \mathrm{rpm}$ and $4^{\circ} \mathrm{C}$ to separate the pore water from the solid phase. The resulting pore water was filtered through $0.45-\mu \mathrm{m}$ Millipore filters, acidified to $\mathrm{pH} 2\left(1 \%\right.$ equivalent volume of ultrapure $\left.\mathrm{HNO}_{3}\right)$ and stored at $4^{\circ} \mathrm{C}$. The sediment samples and the cakes of centrifuged sediment were placed in plastic bags and kept frozen at $-20^{\circ} \mathrm{C}$.

\section{Chemical analysis}

Total dissolved iron and manganese in pore water samples were measured by atomic absorption spectroscopy (AAS), using direct aspiration into an air-acetylene flame Perkin Elmer 4000 device. Sediment aliquots were freeze-dried, homogenized by grinding, and digested with a mixture of acids according to the method described by Rantala and Loring (1977). The digestates were then analyzed by atomic absorption spectroscopy using direct aspiration in a $\mathrm{N}_{2} \mathrm{O}$-acetylene flame (Al, Si, Ca, Mg), an air-acetylene flame (Fe, Mn, Zn) and a pyrolytic graphite furnace equipped with a L'vov multi nucleador a bordo del buque de investigación Mestre Costeiro, en 1998. Se tomaron dos testigos de $30 \mathrm{~cm}$ de longitud ( $\mathrm{E}$ y $\mathrm{G}$ ) en la zona de lodo del Duero y uno de $15 \mathrm{~cm}$ de longitud (J) en la del Miño. Los testigos, que no fueron perturbados por el muestreo, fueron sub-muestreados a bordo en secciones horizontales de $0.5 \mathrm{~cm}$ en los primeros $10 \mathrm{~cm}$ y posteriormente cada $1 \mathrm{~cm}$. Los sedimentos más profundos fueron seccionados en capas de $2 \mathrm{~cm}$. Inmediatamente se separaron alícuotas de $1 \mathrm{~g}$ de sedimento para la determinación de hierro amorfo y sulfuro. Todas las operaciones fueron hechas rápidamente para minimizar cambios resultantes de la exposición al aire. Posteriormente, las muestras se almacenaron en tubos de policarbonato a prueba de fugas, se llenaron completamente dentro de una cámara en presencia de nitrógeno y se mantuvieron en una cámara fría. En el laboratorio, las muestras fueron centrifugadas por $30 \mathrm{~min}$ a 3000 r.p.m. y $4^{\circ} \mathrm{C}$ para separar el agua intersticial de la fase sólida. El agua intersticial fue filtrada a través de filtros Millipore de $0.45 \mu \mathrm{m}$, acidificada a $\mathrm{pH} 2$ (con un volumen equivalente de $\mathrm{HNO}_{3}$ ultrapuro al 1\%) y almacenada a $4^{\circ} \mathrm{C}$. Las muestras de sedimento y las pastillas de sedimento centrifugado fueron colocadas en bolsas de plástico y se mantuvieron congeladas $\mathrm{a}-20^{\circ} \mathrm{C}$.

\section{Análisis químico}

Los contenidos totales de hierro y manganeso disueltos en las muestras de agua intersticial fueron medidos por espectroscopía de absorción atómica (EAA) utilizando aspiración directa a la llama de aire-acetileno en un aparato Perkin Elmer 4000. Las alícuotas de sedimento se congelaron, se molieron para homogeneizarlas y fueron digeridas con una mezcla de ácidos, de acuerdo al método descrito por Rantala y Loring (1977). El material digerido fue analizado posteriormente por espectroscopía de absorción atómica utilizando aspiración directa a la llama de $\mathrm{N}_{2} \mathrm{O}$-acetileno ( $\mathrm{Al}, \mathrm{Si}, \mathrm{Ca}, \mathrm{Mg}$ ), a la flama de aire-acetileno (Fe, $\mathrm{Mn}, \mathrm{Zn}$ ) y en horno de grafito pirolítico equipado con una plataforma $\mathrm{L}^{\prime}$ vov $(\mathrm{Cu}, \mathrm{Pb}$ y $\mathrm{Cd})$. Los errores de precisión fueron: Al 2.3\%, Si 1.6\%, Ca 4.7\%, Mg 3.6\%, Fe $2.3 \%, \mathrm{Mn} 1.0 \%$, Zn $2.0 \%, \mathrm{Cu} 2.0 \%, \mathrm{~Pb} 4.0 \%$ y $\mathrm{Cd} 2.0 \%$ $(P=0.05)$. Las alícuotas de las muestras de sedimento húmedo fueron utilizadas para la determinación de sulfuros. Los sulfuros procedentes de sulfuros volátiles en ácido (AVS) fueron liberados en $\mathrm{HCl} 1 \mathrm{M}$, purgando el sistema con gas nitrógeno y capturando el $\mathrm{H}_{2} \mathrm{~S}$ en una solución de $\mathrm{NaOH} 1 \mathrm{M}$ (Henneke et al., 1991; Madureira et al., 1997). El sulfuro de hidrógeno capturado en la solución básica se determinó por métodos voltamétricos (Luther et al., 1985) utilizando un aparato Methrom equipado con un procesador 693 VA y una base 694 VA. La recuperación de la solución estándar de sulfuro fue del 97\% y el límite de detección del método fue de $0.01 \mu \mathrm{M}$. El Fe y el Zn extraídos simultáneamente, y liberados de los AVS por acidificación del sedimento con $\mathrm{HCl} 1 \mathrm{M}$, fueron analizados directamente por EAA. El Cu, $\mathrm{Pb}$ y Cd extraídos simultáneamente fueron analizados directamente por absorción atómica utilizando un horno de grafito como se describió previamente. 
platform $(\mathrm{Cu}, \mathrm{Pb}$ and $\mathrm{Cd})$. Precision errors were: $\mathrm{Al}, 2.3 \%$; $\mathrm{Si}$, $1.6 \%$; Ca, 4.7\%; Mg, 3.6\%; Fe, 2.3\%; Mn, 1.0\%; Zn, 2.0\%; $\mathrm{Cu}, 2.0 \%$; $\mathrm{Pb}, 4.0 \%$; and $\mathrm{Cd}, 2.0 \%(P=0.05)$. Aliquots of wet sediment samples were used for sulphide determinations. Sulphides from acid volatile sulphide (AVS) were released in $1 \mathrm{M} \mathrm{HCl}$, purging the system with nitrogen gas, and trapping the $\mathrm{H}_{2} \mathrm{~S}$ in $1 \mathrm{M} \mathrm{NaOH}$ solution (Henneke et al., 1991; Madureira et al., 1997). Hydrogen sulphide trapped in the basic solution was determined by voltametric methods (Luther et al., 1985), using a Methrom device equipped with a 693 VA processor and a 694 VA stand. The recovery of the standard sulphide solution was $97 \%$ and the detection limit of the method was $0.01 \mu \mathrm{M}$. The simultaneously extracted iron and zinc, released from the AVS by acidification of the sediment with $1 \mathrm{M} \mathrm{HCl}$, were analyzed directly in the sample by AAS. The simultaneously extracted copper, lead and cadmium were analyzed directly by atomic absorption using a graphite furnace as previously described. The amorphous iron hydroxides were obtained by means of an ascorbate extraction according to the method described by Anschutz et al. (1998), using an aerobic solution at $\mathrm{pH} 8$. Iron concentration in the obtained solution was determined by AAS. International certified standards (MESS-1 and BCSS-1) were used to control the accuracy of the procedure for total metal determinations. The values obtained were not statistically different from the certified standards $(P<0.01)$.

\section{Results and discussion}

\section{Sediment description}

The visual inspection of the collected cores revealed an abundant fraction of fine-grained material, as commonly observed in the muddy patches of the northern Portuguese Shelf (Drago et al., 1998). During the opening procedure, several macrobenthic organisms were found in sub-surface layers of core $G$ (Annelida, Polychaeta, Ophiuroidea and bivalve shells) and core J (high density of small bivalves). Bioturbation was not evident in core $\mathrm{E}$.

\section{Major, minor and trace elemental composition}

The aluminium concentrations and the major, minor and trace metal ratios to $\mathrm{Al}$ of cores $\mathrm{E}, \mathrm{G}$ and $\mathrm{J}$ are presented in table 1. Aluminium concentrations in the three cores were relatively uniform with the depth, generally varying between $6 \%$ and $7 \%$. These values are in agreement with the grain size distribution that indicates a stable deposition of fine-grained material in these muddy patches (Drago, 1995). The Fe/Al ratios ranged also in a narrow interval, with a mean value of $0.36 \pm 0.031$. Values of the $\mathrm{Si} / \mathrm{Al}, \mathrm{Ca} / \mathrm{Al}$ and $\mathrm{Mg} / \mathrm{Al}$ ratios were less uniform, with core $\mathrm{G}$ presenting higher $\mathrm{Si} / \mathrm{Al}$ and lower $\mathrm{Mg} / \mathrm{Al}$ and $\mathrm{Ca} / \mathrm{Al}$ ratios. Moreover, $\mathrm{Ca} / \mathrm{Al}$ and $\mathrm{Mg} / \mathrm{Al}$ ratios in the 1-cm layer were half of the values found in deeper sediments, which indicates that the material is more siliceous and
Los hidróxidos amorfos de hierro fueron obtenidos a partir de una extracción con ascorbato, de acuerdo al método descrito por Anschutz et al. (1998), utilizando una solución aerobia con pH 8. La concentración de hierro en la solución obtenida fue determinada por EAA. Se utilizaron estándares certificados internacionales (MESS-1, BCSS-1) para controlar la precisión del procedimiento en las determinaciones de metales totales. Los valores obtenidos y los de los estándares certificados no fueron estadísticamente diferentes $(P<0.01)$.

\section{Resultados y discusión}

\section{Descripción de sedimentos}

La inspección visual de los testigos muestreados reveló una fracción abundante de material de grano fino como es comúnmente observado en las zonas de lodo de la plataforma norte de Portugal (Drago et al., 1998). Al abrir el testigo se encontraron varios organismos macrobentónicos en las capas sub-superficiales del testigo G (Annelida, Polychaeta, Ophiuroidea, así como conchas de bivalvos) y del testigo J (alta densidad de bivalvos pequeños). Por el contrario, no se observó bioperturbación en el núcleo $\mathrm{E}$.

Composición de elementos mayores, menores y traza

En la tabla 1 se presentan las concentraciones de aluminio, así como las relaciones entre metales mayores, menores y traza con el Al encontradas en los testigos E, G y J. Las concentraciones de aluminio en los tres testigos fueron relativamente uniformes con la profundidad, variando entre 6 y 7\%. Estos valores concuerdan con la distribución de tamaño de grano que indica una sedimentación estable del material de grano fino en estas manchas de lodo (Drago, 1995). Las relaciones Fe/Al también variaron dentro de un margen estrecho con un valor medio de $0.36 \pm 0.031$. Los valores de las relaciones $\mathrm{Si} / \mathrm{Al}, \mathrm{Ca} /$ $\mathrm{Al}$ y $\mathrm{Mg} / \mathrm{Al}$ fueron menos uniformes ya que el núcleo $\mathrm{G}$ presentó una relación alta de $\mathrm{Si} / \mathrm{Al}$, mientras que las relaciones $\mathrm{Mg} / \mathrm{Al}$ y $\mathrm{Ca} / \mathrm{Al}$ fueron bajas. Además, las relaciones $\mathrm{Ca} / \mathrm{Al}$ y $\mathrm{Mg} / \mathrm{Al}$ en la capa de $1 \mathrm{~cm}$ fueron de la mitad de los valores encontrados en los sedimentos más profundos, lo que indica que el material es más silíceo y con un menor contenido de carbonatos. Las razones $\mathrm{Mn} / \mathrm{Al}$ y $\mathrm{Zn} / \mathrm{Al}$ fueron similares en los tres testigos y no variaron considerablemente con la profundidad. El ligero incremento de la relación $\mathrm{Mn} / \mathrm{Al}$ en las primeras capas de $0.5 \mathrm{~cm}$ del núcleo $\mathrm{G}$ puede ser el resultado de procesos diagenéticos (Sundby et al., 1981). También se encontró un gradiente marcado en la relación $\mathrm{Cu} / \mathrm{Al}$ en la capa de $1 \mathrm{~cm}$, lo que puede deberse a la presencia de materia orgánica asentada recientemente y que proviene de la columna de agua. Una disminución pronunciada de la relación $\mathrm{Cu} / \mathrm{Al}$ en el primer centímetro también puede indicar una degradación intensa de la materia orgánica. Esto coincide con el modelo propuesto por Klinkhammer et al. (1982), en el que la mayor parte del Cu que 
Table 1. Aluminium (\%) and Me/Al ratios in sediment cores from the Douro ( $E$ and $G$ ) and Minho $(\mathrm{J})$ muddy patches. Tabla 1. Aluminio (\%) y relaciones Me/Al de los testigos de sedimento de las zonas de lodo del Duero (E y G) y del Miño (J)

\begin{tabular}{|c|c|c|c|c|c|c|c|c|c|c|}
\hline \multicolumn{11}{|c|}{ CORE E } \\
\hline $\begin{array}{l}\text { Layers } \\
(\mathrm{cm})\end{array}$ & $\begin{array}{c}\mathrm{Al} \\
(\%)\end{array}$ & $\mathrm{Si} / \mathrm{Al}$ & $\mathrm{Ca} / \mathrm{Al}$ & $\mathrm{Mg} / \mathrm{Al}$ & $\mathrm{Fe} / \mathrm{Al}$ & $\begin{array}{c}\mathrm{Mn} / \mathrm{Al} \\
\left(10^{-4}\right)\end{array}$ & $\begin{array}{c}\mathrm{Zn} / \mathrm{Al} \\
\left(10^{-4}\right)\end{array}$ & $\begin{array}{c}\mathrm{Pb} / \mathrm{Al} \\
\left(10^{-4}\right)\end{array}$ & $\begin{array}{c}\mathrm{Cu} / \mathrm{Al} \\
\left(10^{-4}\right)\end{array}$ & $\begin{array}{c}\mathrm{Cd} / \mathrm{Al} \\
\left(10^{-4}\right)\end{array}$ \\
\hline 0.25 & 6.2 & 6.1 & 0.55 & 0.19 & 0.40 & 43 & 18 & 5.7 & 2.4 & 0.014 \\
\hline 0.75 & 6.3 & 5.7 & 0.40 & 0.18 & 0.38 & 42 & 18 & 4.7 & 2.0 & 0.012 \\
\hline 1.25 & 5.8 & 6.0 & 0.47 & 0.19 & 0.40 & 45 & 22 & 4.9 & 1.9 & 0.014 \\
\hline 1.75 & 6.2 & 6.0 & 0.35 & 0.16 & 0.34 & 43 & 17 & 4.2 & 2.0 & 0.016 \\
\hline 2.25 & 6.3 & 5.5 & 0.32 & 0.14 & 0.30 & 42 & 23 & 4.8 & 1.9 & 0.011 \\
\hline 2.75 & 6.3 & 5.6 & 0.35 & 0.16 & 0.34 & 42 & 17 & 4.5 & 1.8 & 0.014 \\
\hline 3.25 & 7.0 & 5.7 & 0.35 & 0.15 & 0.33 & 39 & 18 & 4.7 & 1.7 & 0.011 \\
\hline 3.75 & 5.9 & 6.1 & 0.50 & 0.18 & 0.36 & 42 & 20 & 4.5 & 2.7 & 0.017 \\
\hline 4.25 & 6.2 & 6.0 & 0.37 & 0.18 & 0.36 & 39 & 17 & 4.4 & 2.4 & 0.012 \\
\hline 4.75 & 7.4 & 6.2 & 0.37 & 0.15 & 0.31 & 40 & 17 & 4.6 & 2.3 & 0.013 \\
\hline 5.5 & 5.2 & 6.3 & 0.43 & 0.18 & 0.36 & 42 & 19 & 4.7 & 2.8 & 0.012 \\
\hline 6.5 & 6.2 & 6.5 & 0.36 & 0.16 & 0.32 & 43 & 18 & 4.0 & 2.3 & 0.013 \\
\hline 7.5 & 6.3 & 6.1 & 0.42 & 0.18 & 0.38 & 42 & 19 & 4.4 & 2.5 & 0.012 \\
\hline 8.5 & 6.2 & 5.9 & 0.37 & 0.16 & 0.34 & 43 & 19 & 4.3 & 2.5 & 0.012 \\
\hline 9.5 & 6.4 & 5.7 & 0.41 & 0.18 & 0.39 & 40 & 17 & 4.7 & 3.3 & 0.014 \\
\hline 11 & 6.6 & 5.2 & 0.32 & 0.15 & 0.33 & 39 & 17 & 4.2 & 2.6 & 0.014 \\
\hline 13 & 7.0 & 5.0 & 0.35 & 0.18 & 0.36 & 39 & 16 & 4.1 & 3.0 & 0.011 \\
\hline 15 & 7.3 & 4.8 & 0.35 & 0.18 & 0.40 & 37 & 15 & 3.9 & 2.5 & 0.014 \\
\hline 17 & 7.2 & 4.8 & 0.30 & 0.16 & 0.36 & 38 & 15 & 4.1 & 2.6 & 0.014 \\
\hline 19 & 7.4 & 4.6 & 0.27 & 0.15 & 0.31 & 39 & 16 & 4.0 & 2.2 & 0.013 \\
\hline 22 & 6.4 & 5.4 & 0.35 & 0.21 & 0.44 & 38 & 16 & 4.6 & 2.9 & 0.013 \\
\hline 26 & 7.8 & 4.3 & 0.23 & 0.13 & 0.30 & 35 & 14 & 4.0 & 2.5 & 0.012 \\
\hline \multicolumn{11}{|c|}{ CORE G } \\
\hline $\begin{array}{l}\text { Layers } \\
(\mathrm{cm})\end{array}$ & $\begin{array}{l}\mathrm{Al} \\
(\%)\end{array}$ & $\mathrm{Si} / \mathrm{Al}$ & $\mathrm{Ca} / \mathrm{Al}$ & $\mathrm{Mg} / \mathrm{Al}$ & $\mathrm{Fe} / \mathrm{Al}$ & $\begin{array}{c}\mathrm{Mn} / \mathrm{Al} \\
\left(10^{-4}\right)\end{array}$ & $\begin{array}{c}\mathrm{Zn} / \mathrm{Al} \\
\left(10^{-4}\right)\end{array}$ & $\begin{array}{c}\mathrm{Pb} / \mathrm{Al} \\
\left(10^{-4}\right)\end{array}$ & $\begin{array}{c}\mathrm{Cu} / \mathrm{Al} \\
\left(10^{-4}\right)\end{array}$ & $\begin{array}{c}\mathrm{Cd} / \mathrm{Al} \\
\left(10^{-4}\right)\end{array}$ \\
\hline $0-0.5$ & 5.4 & 10.4 & 0.06 & 0.13 & 0.38 & 53 & 21 & 7.2 & 5.0 & 0.035 \\
\hline $0.5-1$ & 5.7 & 10.4 & 0.05 & 0.10 & 0.34 & 47 & 20 & 4.8 & 4.1 & 0.019 \\
\hline $1-1.5$ & 5.9 & 9.9 & 0.09 & 0.21 & 0.34 & 39 & 17 & 6.1 & 2.2 & 0.020 \\
\hline $1.5-2$ & 5.9 & 9.9 & 0.08 & 0.19 & 0.34 & 41 & 18 & 5.9 & 2.2 & 0.012 \\
\hline $2-2.5$ & 6.2 & 9.1 & 0.07 & 0.19 & 0.33 & 37 & 16 & 6.4 & 2.2 & 0.023 \\
\hline $2.5-3$ & 6.2 & 10.4 & 0.07 & 0.20 & 0.34 & 42 & 17 & 5.8 & 2.3 & 0.024 \\
\hline $3-3.5$ & 6.2 & 10.0 & 0.07 & 0.19 & 0.33 & 40 & 17 & 5.8 & 2.1 & 0.016 \\
\hline $3.5-4$ & 5.9 & 9.0 & 0.07 & 0.21 & 0.34 & 41 & 18 & 6.6 & 2.5 & - \\
\hline $4-4.5$ & 5.9 & 9.0 & 0.08 & 0.23 & 0.34 & 39 & 17 & 6.2 & 2.2 & 0.019 \\
\hline $4.5-5$ & 6.5 & 10.0 & 0.07 & 0.21 & 0.31 & 38 & 17 & 5.7 & 2.2 & 0.017 \\
\hline $5-5.5$ & 6.5 & 10.8 & 0.07 & 0.22 & 0.32 & 37 & 17 & 6.8 & 2.5 & 0.017 \\
\hline $5.5-6$ & 6.2 & 11.3 & 0.07 & 0.21 & 0.34 & 41 & 17 & 6.9 & 2.3 & 0.019 \\
\hline $6-6.5$ & 5.7 & 9.9 & 0.07 & 0.26 & 0.34 & 40 & 21 & 6.0 & 2.5 & - \\
\hline $6.5-7$ & 5.9 & 9.5 & 0.07 & 0.25 & 0.36 & 44 & 18 & 8.4 & 2.9 & 0.019 \\
\hline $7-7.5$ & 5.9 & 9.0 & 0.08 & 0.22 & 0.36 & 43 & 18 & 7.9 & 2.7 & 0.019 \\
\hline
\end{tabular}




\begin{tabular}{|c|c|c|c|c|c|c|c|c|c|c|}
\hline $\begin{array}{c}\text { Layers } \\
(\mathrm{cm})\end{array}$ & $\begin{array}{l}\mathrm{Al} \\
(\%)\end{array}$ & $\mathrm{Si} / \mathrm{Al}$ & $\mathrm{Ca} / \mathrm{Al}$ & $\mathrm{Mg} / \mathrm{Al}$ & $\mathrm{Fe} / \mathrm{Al}$ & $\begin{array}{c}\mathrm{Mn} / \mathrm{Al} \\
\left(10^{-4}\right)\end{array}$ & $\begin{array}{c}\mathrm{Zn} / \mathrm{Al} \\
\left(10^{-4}\right)\end{array}$ & $\begin{array}{l}\mathrm{Pb} / \mathrm{Al} \\
\left(10^{-4}\right)\end{array}$ & $\begin{array}{l}\mathrm{Cu} / \mathrm{Al} \\
\left(10^{-4}\right)\end{array}$ & $\begin{array}{c}\mathrm{Cd} / \mathrm{Al} \\
\left(10^{-4}\right)\end{array}$ \\
\hline $7.5-8$ & 6.2 & 9.1 & 0.08 & 0.21 & 0.36 & 43 & 18 & 6.9 & 2.9 & 0.016 \\
\hline $8-8.5$ & 6.2 & 8.6 & 0.08 & 0.24 & 0.37 & 42 & 19 & 7.6 & 3.3 & 0.023 \\
\hline $8.5-9$ & 6.2 & 9.5 & 0.08 & 0.21 & 0.36 & 40 & 21 & 6.9 & 2.9 & 0.039 \\
\hline $9-9.5$ & 6.5 & 9.1 & 0.08 & 0.20 & 0.36 & 38 & 19 & 8.2 & 3.2 & 0.029 \\
\hline $9.5-10$ & 6.7 & 10.4 & 0.08 & 0.20 & 0.37 & 38 & 20 & 8.0 & 3.5 & 0.033 \\
\hline $10-11$ & 6.7 & 11.2 & 0.08 & 0.20 & 0.35 & 38 & 19 & 7.7 & 3.1 & 0.027 \\
\hline $11-12$ & 6.2 & 8.6 & 0.09 & 0.23 & 0.37 & 44 & 19 & 7.1 & 2.8 & 0.042 \\
\hline $13-14$ & 6.5 & 8.7 & 0.08 & 0.21 & 0.36 & 43 & 16 & 8.2 & 2.5 & 0.022 \\
\hline $14-15$ & 6.7 & 7.5 & 0.09 & 0.21 & 0.37 & 43 & 16 & 5.8 & 2.5 & 0.021 \\
\hline $15-17$ & 6.7 & 9.2 & 0.09 & 0.21 & 0.38 & 38 & 17 & 8.5 & 3.2 & 0.023 \\
\hline $17-19$ & 7.6 & 8.2 & 0.10 & 0.21 & 0.38 & 36 & 19 & 6.5 & 2.7 & 0.025 \\
\hline $19-21$ & 7.8 & 7.9 & 0.09 & 0.17 & 0.38 & 37 & 17 & 7.0 & 2.7 & 0.036 \\
\hline $21-23$ & 7.0 & 7.2 & 0.09 & 0.22 & 0.39 & 41 & 18 & 8.4 & 2.8 & 0.034 \\
\hline $23-25$ & 7.0 & 7.2 & 0.09 & 0.21 & 0.38 & 40 & 17 & 6.7 & 3.1 & 0.014 \\
\hline $25-27$ & 7.3 & 7.7 & 0.11 & 0.23 & 0.37 & 36 & 19 & 8.5 & 2.4 & 0.018 \\
\hline \multicolumn{11}{|c|}{ CORE J } \\
\hline $\begin{array}{c}\text { Layers } \\
(\mathrm{cm})\end{array}$ & $\begin{array}{l}\mathrm{Al} \\
(\%)\end{array}$ & $\mathrm{Si} / \mathrm{Al}$ & $\mathrm{Ca} / \mathrm{Al}$ & $\mathrm{Mg} / \mathrm{Al}$ & $\mathrm{Fe} / \mathrm{Al}$ & $\begin{array}{c}\mathrm{Mn} / \mathrm{Al} \\
\left(10^{-4}\right)\end{array}$ & $\begin{array}{l}\mathrm{Zn} / \mathrm{Al} \\
\left(10^{-4}\right)\end{array}$ & $\begin{array}{l}\mathrm{Pb} / \mathrm{Al} \\
\left(10^{-4}\right)\end{array}$ & $\begin{array}{c}\mathrm{Cu} / \mathrm{Al} \\
\left(10^{-4}\right)\end{array}$ & $\begin{array}{l}\mathrm{Cd} / \mathrm{Al} \\
\left(10^{-4}\right)\end{array}$ \\
\hline $0-0.5$ & 6.4 & 3.3 & 0.31 & 0.13 & 0.36 & 45 & 18 & 4.8 & 2.1 & 0.019 \\
\hline $0.5-1$ & 6.5 & 3.4 & 0.33 & 0.13 & 0.36 & 50 & 22 & 5.2 & 2.4 & 0.015 \\
\hline $1-1.5$ & 6.3 & 3.4 & 0.32 & 0.13 & 0.36 & 52 & 24 & 5.2 & 2.3 & 0.016 \\
\hline $1.5-2$ & 6.7 & 3.3 & 0.28 & 0.11 & 0.34 & 48 & 21 & 4.9 & 2.1 & 0.015 \\
\hline $2-2.5$ & 6.5 & 3.4 & 0.33 & 0.12 & 0.38 & 50 & 22 & 5.3 & 2.9 & 0.015 \\
\hline $2.5-3$ & 7.1 & 2.8 & 0.27 & 0.10 & 0.36 & 47 & 19 & 4.8 & 2.3 & 0.042 \\
\hline $3-3.5$ & 6.5 & 3.1 & 0.33 & 0.12 & 0.37 & 50 & 20 & 5.4 & 2.3 & 0.029 \\
\hline $3.5-4$ & 6.5 & 2.8 & 0.33 & 0.10 & 0.33 & 50 & 20 & 5.2 & 2.3 & 0.023 \\
\hline $4-4.5$ & 5.9 & 3.3 & 0.35 & 0.13 & 0.42 & 55 & 22 & 5.7 & 2.5 & 0.076 \\
\hline $4.5-5$ & 7.0 & 2.5 & 0.27 & 0.09 & 0.31 & 47 & 19 & 4.8 & 2.1 & 0.025 \\
\hline $5-5.5$ & 6.6 & 3.1 & 0.31 & 0.12 & 0.38 & 50 & 18 & 5.2 & 2.4 & 0.015 \\
\hline $5.5-6$ & 6.1 & 3.4 & 0.29 & 0.12 & 0.42 & 49 & 20 & 5.4 & 2.3 & 0.016 \\
\hline $6-6.5$ & 6.1 & 3.7 & 0.31 & 0.14 & 0.43 & 53 & 20 & 5.5 & 2.3 & 0.016 \\
\hline $6.5-7$ & 7.6 & 2.4 & 0.21 & 0.06 & 0.32 & 39 & 16 & 4.6 & 2.0 & 0.013 \\
\hline $7-7.5$ & 6.4 & 2.7 & 0.29 & 0.10 & 0.38 & 47 & 17 & 5.3 & 2.5 & 0.030 \\
\hline $7.5-8$ & 7.2 & 2.3 & 0.23 & 0.08 & 0.33 & 45 & 17 & 4.8 & 2.1 & 0.019 \\
\hline $8-8.5$ & 6.6 & 2.4 & 0.26 & 0.10 & 0.37 & 50 & 18 & 5.3 & 2.6 & 0.031 \\
\hline $8.5-9$ & 6.4 & 2.9 & 0.34 & 0.13 & 0.41 & 52 & 17 & 5.4 & 2.5 & 0.016 \\
\hline $9-9.5$ & 6.7 & 2.5 & 0.23 & 0.13 & 0.34 & 48 & 18 & 5.0 & 2.4 & 0.015 \\
\hline $9.5-10$ & 6.6 & 2.8 & 0.30 & 0.13 & 0.39 & 50 & 18 & 5.2 & 2.7 & 0.019 \\
\hline $10-11$ & 7.1 & 2.6 & 0.23 & 0.10 & 0.34 & 45 & 18 & 4.7 & 2.4 & 0.014 \\
\hline $11-12$ & 6.6 & 2.7 & 0.26 & 0.09 & 0.39 & 50 & 18 & 4.9 & 2.6 & 0.023 \\
\hline $12-13$ & 6.2 & 2.7 & 0.35 & 0.17 & 0.44 & 49 & 18 & 5.1 & 2.9 & 0.016 \\
\hline $13-14$ & 7.0 & 2.5 & 0.25 & 0.09 & 0.40 & 47 & 17 & 4.7 & 2.4 & 0.034 \\
\hline
\end{tabular}


contains less carbonates. The $\mathrm{Mn} / \mathrm{Al}$ and $\mathrm{Zn} / \mathrm{Al}$ ratios were similar in the three cores and did not vary considerably with depth. The slight increase of the $\mathrm{Mn} / \mathrm{Al}$ ratio in the first $0.5-\mathrm{cm}$ sediment layer of core $\mathrm{G}$ could be the result of diagenetic processes (Sundby et al., 1981). A steep Cu/Al ratio gradient was also found in the first 1-cm layer, which can be due to the presence of freshly deposited organic matter derived from the water column. The sharp decrease of the $\mathrm{Cu} / \mathrm{Al}$ ratio in the first centimetre may also indicate an intense degradation of organic matter. This agrees with the model proposed by Klinkhammer et al. (1982), with most of the copper arriving to the sea floor being regenerated near the interface and released to the water column. This tendency was not registered in the other cores, probably because of lower carbon input to the sediment at those stations. Whereas $\mathrm{Cd} / \mathrm{Al}$ ratios were almost constant along core $\mathrm{E}$, multiple peaks were recorded in core $\mathrm{G}$ and a broad enhancement, between 2 and $4 \mathrm{~cm}$ depth, was observed in core J. Increase of total cadmium concentration with the sediment depth is reported by Gobeil et al. $(1987,1997)$, and it is attributed to post-mobilization and subsequent precipitation as sulphide. Also, $\mathrm{Pb} / \mathrm{Al}$ ratios were less uniform in core $\mathrm{G}$ and most of their values exceeded those found in cores $\mathrm{E}$ and $\mathrm{J}$.

\section{Dissolved iron and manganese, iron oxides and AVS}

The vertical distributions of total dissolved iron and manganese in sediment pore waters and of AVS in the three cores are shown in figure 2. The manganese and iron profiles in core $\mathrm{E}$ are characterized by regular shapes with maximum values below the sediment-water interface. The concentration peaks indicate the oxide reduction near the surface and the subsequent decreases with the removal of these elements from the solution with depth. The vertical distribution of the iron extracted by the ascorbate method elucidates the depth where iron amorphous oxides are formed (Anschutz et al., 1998). Whereas in core $\mathrm{E}$ iron precipitated just below the sedimentwater interface, the iron oxide peak in core $G$ was formed in the sub-surface layers where several maxima and minima of dissolved iron are present. The values of iron oxides in core $\mathrm{J}$ were uniform with depth. The AVS levels increased with depth as dissolved iron in pore waters decreased, which confirms the removal of iron from solution. Manganese and iron in pore waters of cores $G$ and $J$ showed irregular shapes with successive maxima and minima. The manganese maximum occurred in sub-surface layers and iron increased in deeper sediment layers, between 5 and $13 \mathrm{~cm}$. Whereas manganese concentrations were similar in the three cores, iron in pore waters of core $\mathrm{E}$ exceeded ten times the values found in core $\mathrm{G}$. The AVS values were low $\left(<0.01 \mu \mathrm{mol} \mathrm{g}{ }^{-1}\right)$ in the first $5 \mathrm{~cm}$ and then increased, showing multiple peaks in core G. Levels of AVS were uniformly low $\left(<0.01 \mu \mathrm{mol} \mathrm{g}^{-1}\right)$ along core J, except in two layers, around the 8 and $13 \mathrm{~cm}$ depths, where concentrations were ten times lower than values recorded in cores $\mathrm{G}$ and $\mathrm{E}$. llega al piso oceánico es regenerado cerca de la interfase y es liberado a la columna de agua. No se registró esta tendencia en los otros testigos probablemente como consecuencia de un bajo aporte de carbono a los sedimentos de esas estaciones. Mientras que las relaciones $\mathrm{Cd} / \mathrm{Al}$ siempre fueron constantes a lo largo del testigo $\mathrm{E}$, en el testigo $\mathrm{G}$ se registraron múltiples máximos, a la vez que en el testigo J se observó un enriquecimiento considerable entre los 2 y los $4 \mathrm{~cm}$ de profundidad. El incremento en la concentración de $\mathrm{Cd}$ total con la profundidad del sedimento ha sido descrito por Gobeil et al. (1987, 1997), y se ha atribuido a la post-movilización y subsecuente precipitación en forma de sulfuro. De igual forma, las razones $\mathrm{Pb} / \mathrm{Al}$ fueron menos uniformes en el testigo G, y la mayoría de sus valores excedieron a los encontrados en los testigos $\mathrm{E}$ y J.

Hierro y manganeso disueltos, óxidos de hierro y sulfuros volátiles en ácido (AVS)

La distribución vertical de los contenidos totales de hierro y manganeso disueltos en el agua intersticial del sedimento, así como de los AVS en los tres testigos se muestran en la figura 2. Los perfiles de hierro y manganeso en el testigo E se caracterizan por una forma regular con valores máximos por debajo de la interfase sedimento-agua. Los máximos de concentración indican procesos de óxido-reducción cerca de la superficie, lo que conlleva a la remoción de estos elementos de la solución al incrementarse la profundidad. La distribución vertical del hierro extraído por el método del ascorbato, sugiere la profundidad donde se forman los óxidos amorfos de hierro (Anschutz et al., 1998). Mientras que en el testigo E el hierro se precipitó por debajo de la interfase sedimento-agua, en el testigo $\mathrm{G}$ el máximo de óxido de hierro apareció en las capas sub-superficiales donde se presentan varios valores máximos y mínimos de hierro disuelto. Los valores de óxidos de hierro en el testigo $\mathrm{J}$ fueron uniformes con la profundidad. Los valores de AVS se incrementaron con la profundidad, mientras que el hierro disuelto en agua intersticial disminuyó, lo que confirma la remoción del hierro de la solución. El manganeso y el hierro en el agua intersticial de los testigos $\mathrm{G}$ y $\mathrm{J}$ mostraron distribuciones irregulares con máximos y mínimos sucesivos. El máximo de manganeso se presentó en las capas subsuperficiales y el hierro se incrementó en las capas más profundas del sedimento, entre los $5 \mathrm{y} \operatorname{los} 13 \mathrm{~cm}$. Aunque las concentraciones de manganeso fueron similares en los tres testigos, el hierro en el agua intersticial del núcleo E excedió diez veces el valor encontrado en el núcleo G. Los valores de AVS fueron bajos $\left(<0.01 \mu \mathrm{mol} \mathrm{g}^{-1}\right)$ en los primeros cinco centímetros y posteriormente se incrementaron, mostrando múltiples picos máximos en el núcleo G. Los niveles de AVS fueron uniformemente bajos $\left(<0.01 \mu \mathrm{mol} \mathrm{g}^{-1}\right)$ a lo largo del núcleo $\mathrm{J}$, a excepción de dos capas alrededor de los 8 y los $13 \mathrm{~cm}$ de profundidad donde las concentraciones fueron diez veces más bajas que los valores registrados en los testigos $\mathrm{G}$ y E. 

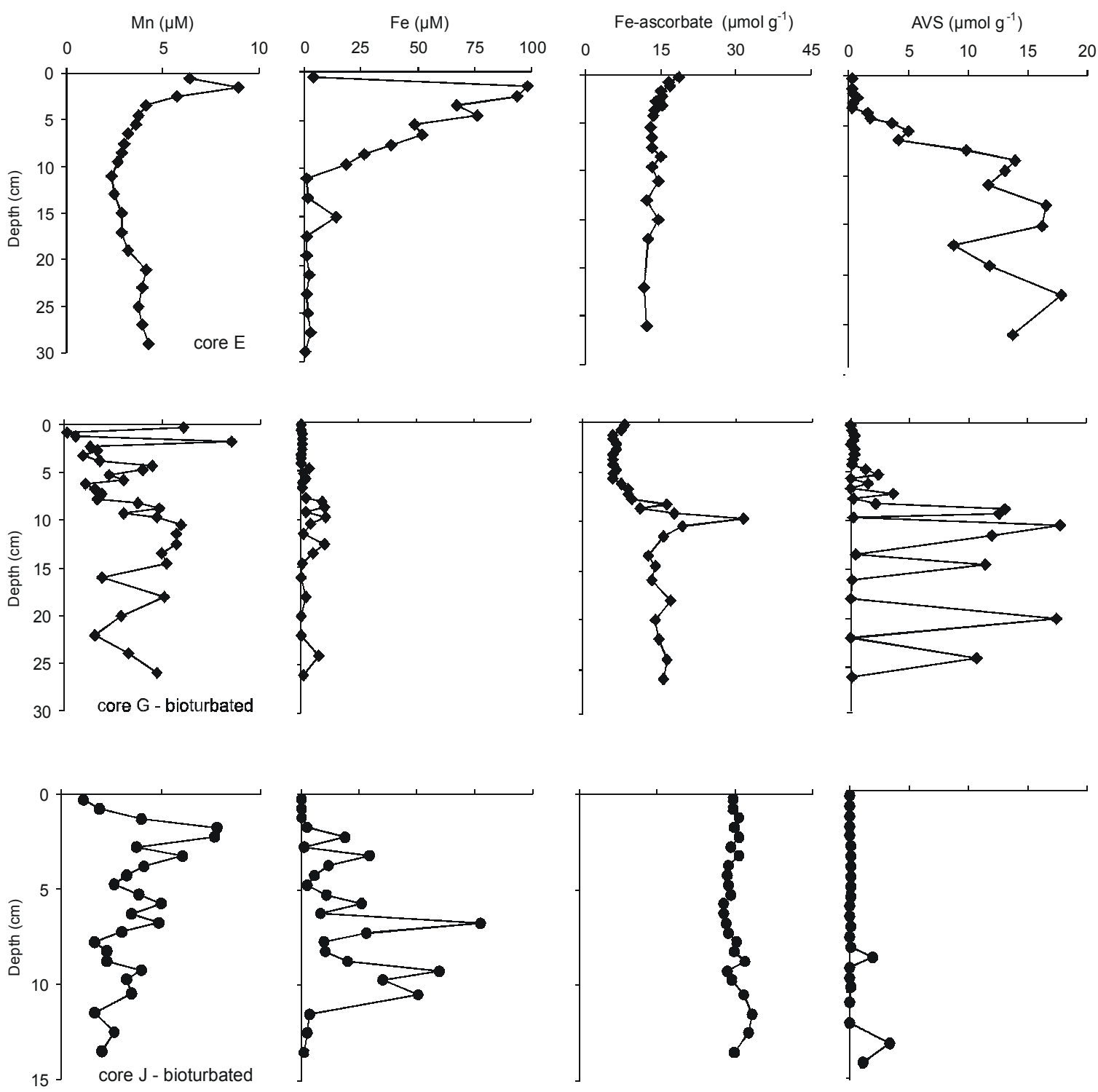

Figure 2. Vertical profiles of dissolved $\mathrm{Mn}(\mu \mathrm{M})$, dissolved $\mathrm{Fe}(\mu \mathrm{M})$, amorphous iron oxides $\left(\mu \mathrm{mol} \mathrm{g}^{-1}\right)$ and acid volatile sulphides (AVS, $\mu \mathrm{mol} \mathrm{g}^{-1}$ ) in sediment cores $\mathrm{E}, \mathrm{G}$ and $\mathrm{J}$.

Figura 2. Perfiles verticales de Mn disuelto $(\mu \mathrm{M})$, Fe disuelto $(\mu \mathrm{M})$, óxidos de hierro amorfos $\left(\mu \mathrm{mol} \mathrm{g}^{-1}\right)$ y sulfuros volátiles en ácidos (AVS, $\mu \mathrm{mol} \mathrm{g}^{-1}$ ) en los testigos de sedimento $\mathrm{E}, \mathrm{G}$ y $\mathrm{J}$

Simultaneously extracted metals with AVS

The concentrations of iron, zinc, copper, lead and cadmium extracted from the sediments with a $1 \mathrm{M} \mathrm{HCl}$ solution showed pronounced increases below $1 \mathrm{~cm}$ in core $\mathrm{E}$ and below $10 \mathrm{~cm}$ in core $\mathrm{G}$ (fig. 3). In core E, concentrations of all the analyzed metals increased sharply below $1 \mathrm{~cm}$ as iron is removed from pore waters, and exhibited maximum values between 2 and $4 \mathrm{~cm}$, where AVS show their first increases in the sediment column ( 0.4 to $\left.1.9 \mu \mathrm{mol} \mathrm{g}^{-1}\right)$. The trace metal maxima did not occur at the same depth and the shape of their curves varied slightly from metal to metal. Iron, zinc and copper showed maximum values at the same depth, while lead and cadmium

\section{Metales extraídos simultáneamente con los AVS}

Las concentraciones de hierro, zinc, cobre, plomo y cadmio extraídos de los sedimentos con una solución de $\mathrm{HCl} 1 \mathrm{M}$, mostraron incrementos pronunciados por debajo de $1 \mathrm{~cm}$ de profundidad en el testigo $\mathrm{E}$ y por debajo de $l o s \quad 10 \mathrm{~cm}$ en el testigo G (fig. 3). En el testigo E, las concentraciones de todos los metales analizados se incrementaron de manera muy marcada por debajo de $1 \mathrm{~cm}$ de profundidad, conforme el hierro es removido del agua intersticial, y mostraron los valores más altos entre los 2 y $4 \mathrm{~cm}$ de profundidad, donde los AVS presentaron sus primeros incrementos en la columna de sedimento $\left(0.4\right.$ a $\left.1.9 \mu \mathrm{mol} \mathrm{g}^{-1}\right)$. El valor máximo para los metales traza no 

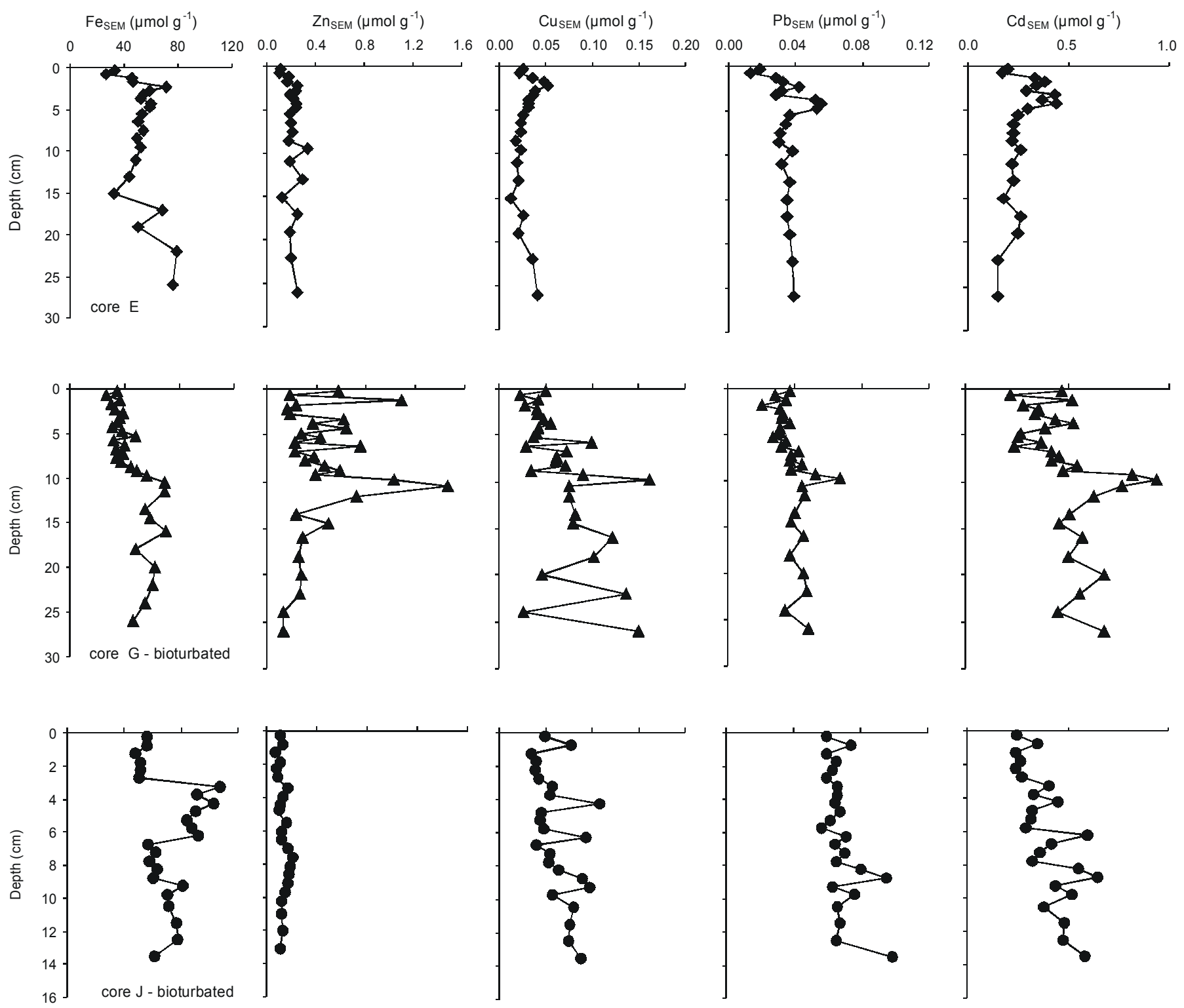

Figure 3. Vertical profiles of $\mathrm{Fe}, \mathrm{Zn}, \mathrm{Cu}, \mathrm{Pb}$ and $\mathrm{Cd}\left(\mu \mathrm{mol} \mathrm{g}{ }^{-1}\right)$ simultaneously extracted with acid volatile sulphides (AVS) in sediments cores $\mathrm{E}$, $\mathrm{G}$ and $\mathrm{J}$.

Figura 3. Perfiles verticales de $\mathrm{Fe}, \mathrm{Zn}, \mathrm{Cu}, \mathrm{Pb}$ y $\mathrm{Cd}\left(\mu \mathrm{mol} \mathrm{g}^{-1}\right)$ extraídos simultáneamente con los sulfuros volátiles en ácidos (AVS) de los testigos de sedimento $E, G$ y J.

had a second peak right below them. Peaks of extractable copper, lead and cadmium are sharp and values were two to three times those found at the sediment surface. The iron maximum was broader in all the cores analyzed. In core $\mathrm{G}$, the maximum was registered deeper in the sediment, just below the amorphous iron oxide peak and in the zone of irregular AVS concentrations. A sharp increase of zinc was recorded at the same sediment depth, while maximum values of copper, lead and cadmium occurred $0.5 \mathrm{~cm}$ above. The profiles in core $\mathrm{J}$ were not clear and only iron showed a broad maximum where the levels of AVS varied abruptly (fig. 3). Maximum concentrations of trace metals in core $\mathrm{G}$ exceeded largely the values found in the other two cores analyzed. fue registrado a la misma profundidad y la forma de las curvas varió muy poco entre los diferentes metales. El hierro, el zinc y el cobre mostraron valores máximos a la misma profundidad, mientras que el plomo y el cadmio presentaron un segundo pico máximo inmediatamente por debajo de aquellos. Los máximos de cobre, plomo y cadmio extraídos son muy marcados, y sus valores fueron de 2 a 3 veces mayores que los encontrados en los sedimentos superficiales. El valor máximo para el hierro fue más amplio en todos los testigos analizados. En el testigo G, el máximo fue registrado a mayor profundidad, justo por debajo del pico mostrado por los óxidos de hierro amorfos y en la zona donde se presentaron concentraciones irregulares de AVS. A esta misma profundidad del sedimento 


\section{Mobilization of trace metals in oxic/anoxic interfaces}

Where bioturbation was not visible (core E) the profiles of copper, lead and cadmium extracted with a $1 \mathrm{M} \mathrm{HCl}$ solution are consistent with the precipitation of downward diffusing dissolved metals. This implies that trace metals remobilize in the oxic surface layer of the muddy patches. Several works have proposed the regeneration of most transition metals near the sediment-water interface (Klinkhammer et al., 1982; Shaw et al., 1990). The release of cadmium in the oxic layers of St. Lawrence sediments and its downward diffusion influences the sediment depth distribution (Gobeil et al., 1997). Lead profiles both in pore waters and the extractable solid fraction evidence its remobilization inside the sediments (Gobeil and Silverberg, 1989). The close correspondence found in our study between the iron, copper, lead, cadmium and AVS distributions is in complete agreement with sulphide precipitation. The precipitation of cadmium and lead slightly below copper sulphides could be interpreted as a higher availability in pore waters, or diffusion to the layers where metals meet dissolved sulphide produced deeper by sulphate reduction. Because only $18 \%$ of copper and $30 \%$ of cadmium were involved in this diagenetic process the total metal concentrations were not affected substantially.

The contrast between the trace metal distributions in cores $\mathrm{G}$ and $\mathrm{E}$ is striking. The peaks of reactive trace metals in the core bioturbated by macrobenthic organisms (G) occurred deeper in the sediments where AVS presented their first maximum-minimum oscillation with depth. This distribution pattern is extended at least until $30 \mathrm{~cm}$ depth. A thicker benthic layer and irrigation mean that dissolved oxygen is pumped deeper into the anoxic zone creating several oxic/anoxic interfaces. The irregular AVS profile and the sharp peak of iron oxides corroborate the dramatic changes occurred inside the sediments. Because inhabitant organisms create these interfaces, this situation is probably in non-steady-state conditions. The net result of several redox boundaries is to mobilize trace metals between oxic/suboxic zones and layers where sulphide is present in a larger sediment volume. This mobilization explains the higher quantities of zinc, copper and cadmium associated with monosulphides in the bioturbated core $\mathrm{G}$. Presumably the intensity of these exchanges is enough to influence the total metal distribution, in the solid fraction of the sediments.

\section{Conclusions}

The trace metal distribution recorded in sediment cores from muddy patches of the northern Portuguese Shelf points to the importance of bioturbation on the redistribution and fractionation of metals in coastal sediments. Irrigation and sediment bioturbation causes irregularities in metal fractionation as response to inter-layers of metal oxides and sulphides. se registró un notable enriquecimiento de zinc, mientras que los valores máximos del cobre, plomo y cadmio se ubicaron $0.5 \mathrm{~cm}$ arriba de este. Los perfiles en el testigo $\mathrm{J}$ no fueron claros y sólo el hierro mostró un máximo amplio donde los niveles de AVS variaron abruptamente (fig. 3). Las concentraciones máximas de los metales traza en el testigo $\mathrm{G}$ excedieron en gran medida los valores encontrados en los otros dos testigos analizados.

\section{Movilización de metales traza en interfases óxico-anóxicas}

En donde no fue evidente la bioperturbación (núcleo E), los perfiles de cobre, plomo y cadmio extraídos con $\mathrm{HCl} 1 \mathrm{M}$ concuerdan con la precipitación por difusión descendente de los metales disueltos. Esto implica que los metales traza se removilizan en la capa superficial óxica de las zonas de lodo. Varios trabajos han propuesto la regeneración de la mayoría de los metales de transición cerca de la interfase sedimento-agua (Klinkhammer et al., 1982; Shaw et al., 1990). La liberación de cadmio en las capas óxicas de los sedimentos de St. Lawrence y la difusión descendente influencian la distribución en los sedimentos profundos (Gobeil et al., 1997). Los perfiles de plomo, tanto en agua intersticial como en las fracciones sólidas extraíbles, dan evidencia de su removilización en los sedimentos (Gobeil y Silverberg, 1989). La correspondencia cercana encontrada en nuestro estudio entre las distribuciones de $\mathrm{Fe}, \mathrm{Cu}, \mathrm{Pb}, \mathrm{Cd}$ y AVS, concuerda totalmente con la precipitación de los sulfuros. La precipitación del cadmio y del plomo un poco más abajo que la de los sulfuros de cobre podría interpretarse como una mayor disponibilidad en el agua intersticial, o como difusión hacia las capas en donde los metales encuentran sulfuros disueltos que son producidos en zonas más profundas por la reducción del sulfato. Dado que sólo el $18 \%$ del $\mathrm{Cu}$ y el $30 \%$ del $\mathrm{Cd}$ estuvieron involucrados en este proceso diagenético, las concentraciones de metales totales no se vieron afectadas de manera sustancial.

El contraste entre las distribuciones de los metales traza en los testigos $\mathrm{G}$ y $\mathrm{E}$ es sorprendente. Los picos de metales traza reactivos en el testigo con bioperturbación por organismos macrobénticos $(\mathrm{G})$ ocurrieron en zonas más profundas de los sedimentos donde los AVS presentaron su primera oscilación de máximo a mínimo con la profundidad. Este patrón de distribución se extiende al menos hasta los $30 \mathrm{~cm}$ de profundidad. Una capa bentónica más gruesa e irrigación significan que el oxígeno disuelto es bombeado hacia zonas anóxicas profundas, originando de esta manera interfases óxico-anóxicas. Tanto el perfil irregular de AVS como el máximo pronunciado de los óxidos de hierro corroboran los dramáticos cambios que ocurren en el interior de los sedimentos. Debido a que los organismos que los habitan crean estas interfases, es probable que esta situación ocurra bajo condiciones no estables. El resultado neto de la existencia de varias fronteras redox es la movilización de los metales traza entre zonas óxicas/subóxicas y capas donde los sulfuros se presentan en un gran volumen del sedimento. Esta movilización explica las grandes cantidades de 


\section{Acknowledgements}

The authors wish to thank Teresa Drago for the description of the cores.

\section{References}

Aller, R.C. (1977). Diagenetic processes near the sediment-water interface of Long Island Sound. II. Fe and Mn. Adv. Geophys., 22: 351-415.

Aller, R.C. (1994). The sedimentary Mn cycle in Long Island Sound: Its role as intermediate oxidation and the influence of bioturbation, $\mathrm{O}_{2}$, and $\mathrm{C}_{\text {org }}$ flux on diagenetic reaction balances. J. Mar. Res., 52: 259-295.

Anschutz, A., Zhong, S., Sundby, B., Mucci, A. and Gobeil, C. (1998). Burial efficiency of phosphorus and the geochemistry of iron in continental margin sediments. Limnol. Oceanogr., 43(1): $53-64$.

Araújo, M.F., Dias, J.M.A. and Jouanneau, J.-M. (1994). Chemical characterization of the main fine sedimentary deposit at the northwestern Portuguese shelf. GAIA, 9: 59-65.

Barbosa, T., Araújo, M.F.D., Jouanneau, J.-M., Gouveia, M.A., Weber, O. and Dias, J.M.A. (1999). Geochemistry of sediments from the Portuguese shelf. Actas do II Congresso Ibérico de Geoquímica, XI Semana da Geoquímica, Lisbon, Portugal, 14-17 June, pp. 437-440.

Berner, R.A. (1980). Early Diagenesis: A Theoretical Approach. Princeton Univ. Press, New Jersey, 241 pp.

Brügmann, L., Hallberg, R., Larsson, C. and Löffler, A. (1998). Trace metal speciation in sea and pore water of the Gotland Deep, Baltic Sea, 1994. Appl. Geochem., 13: 359-368.

Dias, J.M.A. and Nittrouer, C.A. (1984). Continental shelf sediments of northern Portugal. Cont. Shelf Res., 3(2): 147-165.

Drago, T. (1995). La vasiére ouest-Douro sur la plataforme continentale nord-portugaise. Rôle, fonctionment, evolution. $\mathrm{Ph} . \mathrm{D}$. thesis, Univ. Bordeaux I (unpublished).

Drago, T., Oliveira, A., Magalhães, F., Cascalho, J., Jouanneau, J.-M. and Vitorino, J. (1998). Some evidences of northward fine sediment transport in the northern Portuguese continental shelf. Oceanol. Acta, 21(2): 223-231.

Froelich, P.N., Klinkhammer, G.P., Bender, M.L., Luedtke, N.A., Heath, G.R., Cullen, D., Dauphin, P., Hammond, D., Hartman, B. and Maynard, V. (1979). Early oxidation of organic matter in pelagic sediments of the eastern equatorial Atlantic: Suboxic diagenesis. Geochim. Cosmochim. Acta, 43: 1075-1090.

Gobeil, C. and Silverberg, N. (1989). Early diagenesis of lead in Laurentian Trough sediments. Geochim. Cosmochim. Acta, 53: 1889-1895.

Gobeil, C., Silverberg, N., Sundby, B. and Cossa, D. (1987). Cadmium diagenesis in Laurentian Trough sediments. Geochim.Cosmochim. Acta, 51: 589-596.

Gobeil, C., Macdonald, R.W. and Sundby, B. (1997). Diagenetic separation of cadmiun and manganese in suboxic continental margin sediments. Geochim. Cosmochim. Acta, 21: 4647-4654.

Henneke, E., Luther, G.W. and DeLange, G. J. (1991). Determination of inorganic sulphur speciation with polarographic techniques:
$\mathrm{Zn}, \mathrm{Cu}$ y $\mathrm{Cd}$ asociadas con monosulfuros en el testigo bioperturbado $\mathrm{G}$.

Presumiblemente, la intensidad de estos cambios es suficiente para influenciar la distribución total de los metales en la fracción sólida del sedimento.

\section{Conclusiones}

La distribución de metales traza registrada en testigos de sedimento de zonas de lodo de la plataforma norte de Portugal señala la importancia de la bioperturbación en la redistribución y el fraccionamiento de los metales en sedimentos costeros. La irrigación y la bioperturbación del sedimento originan irregularidades en el fraccionamiento de los metales como respuesta a inter-capas de óxidos de metales y sulfuros.

\section{Agradecimientos}

Los autores agradecen a Teresa Drago por la descripción de los testigos.

Traducido al español por Adrián R. López-González.

Some preliminary results for recent hypersaline anoxic sediments. Mar. Geol., 100: 115-123.

Klinkhammer, G., Heggie, D.T. and Graham, D.W. (1982). Metal diagenesis in oxic marine sediments. Earth Planetary Sci. Lett., 61:

211-219.

Luther, G.W., Giblin, A.E. and Varsolona, R. (1985). Polarographic analysis of sulfur species in marine porewaters. Limnol. Oceanogr., 30: 727-736.

Madureira, M.J., Vale, C. and Simões Gonçalves, M.L., (1997). Effect of plants on sulphur geochemistry in the Tagus salt-marshes sediments. Mar. Chem., 58: 27-37.

Pedersen, T.F., Vogel, J.S. and Southon, J.R. (1986). Copper and manganese in hemipelagic sediments at $21^{\circ} \mathrm{N}$, East Pacific Rise: Diagenetic contrast. Geochim. Cosmochim. Acta, 50: 2019-2031.

Rantala, R.T.T. and Loring, D.H. (1977). A rapid determination off 10 elements in marine suspended particulate matter by atomic absortion. Atom. Absorpt. Newslett., 16: 51-52.

Shaw, T.J., Gieskes, J.M. and Jahnke, R.A. (1990). Early diagenesis in differing depositional environments: The response of transition metals in pore water. Geochim. Cosmochim. Acta, 54: 12331246.

Sundby, B., Silverberg, N. and Chesslet, R. (1981). Pathways of manganese in an open estuarine system. Geochim. Cosmochim. Acta, 45: 293-307.

Thamdrup, B., Fossing, H. and Jørgensen, B. B. (1994). Manganese, iron, and sulfur cycling in a coastal marine sediment, Aarhus Bay, Denmark. Geochim. Cosmochim. Acta, 58(23): 5115-5129.

Thomson, H., Higgs, N.C., Croudace, I.W., Colley, S. and Hydes D.J. (1993). Redox zonation of elements at an oxic/postoxic boundary in deep sea sediments. Geochim. Cosmochim. Acta, 57: 579-595. 